Paras Ram and Anil Kumar*

\title{
Analysis of Heat Transfer and Lifting Force in a Ferro-Nanofluid Based Porous Inclined Slider Bearing with Slip Conditions
}

https://doi.org/10.1515/nleng-2018-0014

Received November11, 2017; revised February 20, 2018; accepted March 25, 2018.

\begin{abstract}
Thermal effects have been investigated in a porous inclined slider bearing together with the slip boundary conditions. Using Jenkins model, the governing system of equations pertaining to the flow is solved analytically to yield the various bearing characteristics. The expressions for mean temperature, pressure and the lifting force (load carrying capacity) have been derived as a function of slip, magnetic, permeability, material and thermal parameters. Furthermore, the term pertaining to the co-rotational derivative of magnetization is expected to influence the lifting force significantly. Therefore its effect on the bearing characteristics is also considered. The lubricant is assumed to be incompressible, and its viscosity varies exponentially with the temperature. The behavior of mean temperature with other bearing characteristics across the fluid film thickness has also been investigated. The variations in the lifting force and mean temperature w.r.t. various bearing parameters have been analyzed graphically.
\end{abstract}

Keywords: Jenkins model, Magnetic fluid, Porous inclined slider bearing, Slip velocity, Mean temperature, Thermal boundary layer

\section{Introduction}

Nanofluid is a homogeneous mixture of liquid, i.e. the base fluids like water, oil or ethylene-glycol and particles of solids like copper $(\mathrm{Cu})$, silver $(\mathrm{Ag})$, iron $(\mathrm{Fe})$, etc. with size less than 100nm. The Ferrofluid (FF) belongs to a special

\footnotetext{
Paras Ram, Department of Mathematics, National Institute of Technology, Kurukshetra, Haryana, India,

E-mail: parasram_nit@yahoo.co.in

*Corresponding Author: Anil Kumar, Department of Mathematics, National Institute of Technology, Kurukshetra, Haryana, India, E-mail: anilkumar6150013@gmail.com
}

class of synthetic fluids which undergo significant variation in flow behavior due to an external magnetic field. Ferrofluids are prepared by suspending magnetic solid particles in non-magnetic and non-conducting liquids such as di-esters, kerosene, hydrocarbons, fluorocarbons, water, etc. A fluid with solid Ferro particles of nanosize suspended in it is known as Ferro-nanofluid. These solid particles are in Brownian motion and migrate from higher temperature to the lower temperature region due to Brownian diffusion and thermophoresis forces [1]. In engineering, Fluid flow and Heat transfer phenomenon is a topic of keen interest. It is well known that thermal conductivity of nanofluid is higher than the base fluid because of the high conductivity of suspended particles [2]. Numerica [3] and experimental [4] study on forced and mixed convection proved that the transfer of heat is more in nanofluid as compared with the ordinary fluid. Moreover, the heat transfer rate increases with increase in the volume fraction [5]. In a study, A. Karimipour concluded that $2 \%$ volume fraction of $\mathrm{Cu}$ or Ag led to an increase of $30 \%$ (approx.) in the Nusselt number [6].

In recent years, many researchers have focused their work in examining the lubrication behavior of various Newtonian and non-Newtonian fluids. According to the classical theory of hydrodynamic lubrication, the lubricant was implicitly assumed as a Newtonian viscous fluid. But in modern industrial technology, the fluids like pulps, emulsions, molten plastics, slurries, grease etc. exhibiting non-Newtonian behavior, are also widely used as a lubricant. Therefore, the non-Newtonian behavior of lubricant is also of considerable interest. In order to prevent the viscosity variation with heat, the particles of insoluble solids having different material properties can be used as suspensions in the lubricant to enhance the viscosity index. Such type of lubricants exhibits the nonNewtonian behavior [7]. In lubricants, a considerable variation in the viscosity can be observed due to the temperature generated by frictional heating inside the film. Consequently, a mathematical mapping between the viscosity and the temperature exists [8]. In rotating disk problems, it is a well-reported fact that the heat transfer rate in- 
creases with the increase in the magnetic rotation parameter and the Prandtl number while the trend is opposite with porous permeability parameter, heat generation/ absorption parameter and Eckert number $[9,10]$. In free convective flow, the enhancement in the magnetic parameter leads to decrease in the velocity, i.e., the magnetic force behaves like a retarding force [11]. Dual nature of temperature profile has been noticed in an unsteady convective flow of magnetic fluid in the presence of stretching effects [12]. Due to long-term stability and high thermal conductivity, magnetic fluids have attracted the researchers working on problems pertaining to various kinds of geometries like cylinder, rotating disk, sliding planar motion table, helical pipes, etc. [13-17].

In bearings, magnetic fluids or Ferrofluid (FF) [18] are known to play an important role to enhance the load carrying capacity and heat transfer. Depending on the requirements, the researchers usually adopt three basic magnetic fluid flow models, i.e. Jenkins model [19-23], Shliomis model [24, 25] and Neuringer-Rosensweig model (N-R model) [26, 27]. For higher loads, Shliomis model is suitable whereas N-R and Jenkins models are preferred for lower and moderate loads. Shliomis model accounts for the rotation of magnetic particles, their volume fraction and magnetic moments. The N-R model modifies the pressure while Jenkins model modifies both pressure and velocity of the magnetic fluid through an additional term pertaining to the co-rotational derivative of magnetization, which is missing in N-R model. This additional term takes care of velocity effects in the fluid flow. In a bearing design, the boundary slip and the surface texture are two important aspects and have similar effects on its tribological performance [28]. According to Navier boundary condition, the slip velocity is proportional to the rate of surface shear [29]. The position and the size of surface texture or slip region may either enhance or impair the lifting force of a bearing. Therefore, only a properly designed slip surface or surface texture can improve the bearing performance in terms of the lifting force [30, 31].

In the present problem, the work by Ram et al. [21] has been extended to analyze the rate of heat transfer by introducing the thermal effects in the porous inclined slider bearing together with the slip conditions. The term pertaining to the co-rotational derivative of magnetization has also been taken into account because of its significant impact on the bearing characteristics, and it was ignored by Singh and Ahmad [20]. The expressions for mean temperature, pressure and the lifting force (load capacity) have been derived as a function of slip, magnetic, permeability, material and thermal parameters. Using Simpson's $1 / 3^{\text {rd }}$ rule, the values of the mean temperature and the lifting force have been computed for random values of various non-dimensional parameters by dividing the range of integration into 100 equal parts. The behavior of mean temperature has been investigated across the length of the bearing to examine the rate of heat transfer. Under the influence of material and magnetic parameter, the effects of slip and permeability on the lifting force have also been examined.

\section{Formulation of the Problem}

The governing equations pertaining to the flow of Ferrofluid in vector notation due to Ram and Verma [19] in the domain $D=\{(x, y): 0 \leq x \leq L$ and $0 \leq y \leq h\}$ are:

$$
\begin{gathered}
\rho\left[\frac{\partial q}{\partial t}+(q . \nabla) q\right]=-\nabla p+\mu \nabla^{2} q+\mu_{0}(M . \nabla) H \\
+\rho \alpha^{2} \nabla \times\left(\frac{\tilde{M}}{M} \times M^{\star}\right) \\
\nabla \cdot q=0 ; \\
\nabla \times H=0, H=-\nabla \phi ; \\
\nabla \cdot(H+4 \pi M)=0 ;
\end{gathered}
$$

and

$$
\beta \frac{D^{2} \tilde{M}}{D t^{2}}=-4 \pi p \frac{M_{s}}{\chi_{0}} \frac{\tilde{M}}{M_{s}-M}-\frac{2 \alpha^{2}}{M} M^{\star}+H
$$

where

$$
M^{\star}=\frac{D \tilde{M}}{D t}+\frac{1}{2}(\nabla \times q) \times \tilde{M}
$$

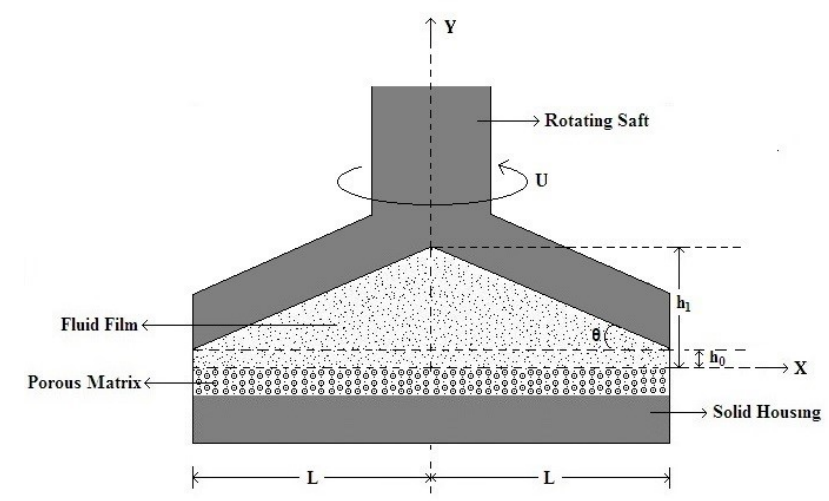

Fig. 1: Porous inclined slider bearing with ferrofluid lubrication 
The equation of continuity in the porous region is given by

$$
\frac{\partial \bar{u}}{\partial x}+\frac{\partial \bar{v}}{\partial y}=0
$$

The simplified form of Eqs. (1) - (6) are given as:

$$
\begin{gathered}
\mu \frac{\partial^{2} u}{\partial y^{2}}-\frac{\partial}{\partial x}\left(p-\frac{\mu_{0} \bar{\mu}}{2} H^{2}\right)-\frac{\rho \alpha^{2} \bar{\mu}}{2} \frac{\partial}{\partial y}\left(H \frac{\partial u}{\partial y}\right)=0 \\
\frac{\partial u}{\partial x}+\frac{\partial v}{\partial y}=0 ; \\
-\frac{\partial}{\partial y}\left(p-\frac{\mu_{0} \bar{\mu}}{2} H^{2}\right)+\frac{\rho \alpha^{2} \bar{\mu}}{2} \frac{\partial}{\partial x}\left(H \frac{\partial u}{\partial y}\right)=0 \\
\bar{u}=-\frac{k}{\mu} \frac{\partial}{\partial x}\left(\bar{p}-\frac{\mu_{0} \bar{\mu}}{2} H^{2}\right)-\frac{\rho \alpha^{2} \bar{\mu}}{2} \frac{k}{\mu} \frac{\partial}{\partial y}\left(H \frac{\partial u}{\partial y}\right) ; \\
\bar{v}=-\frac{k}{\mu} \frac{\partial}{\partial y}\left(\bar{p}-\frac{\mu_{0} \bar{\mu}}{2} H^{2}\right)+\frac{\rho \alpha^{2} \bar{\mu}}{2} \frac{k}{\mu} \frac{\partial}{\partial x}\left(H \frac{\partial u}{\partial y}\right) ;
\end{gathered}
$$

Using (11) and (12) into (7), we obtain

$$
\frac{\partial^{2}}{\partial x^{2}}\left(p-\frac{\mu_{0} \bar{\mu}}{2} H^{2}\right)+\frac{\partial^{2}}{\partial y^{2}}\left(p-\frac{\mu_{0} \bar{\mu}}{2} H^{2}\right)=0
$$

The relevant boundary condition for the velocity field in the lubrication region is

$$
u=U \text { at } y=h \text {, }
$$

where, $U$ is the uniform sliding velocity component along the $\mathrm{x}$-axis and $h$ is the dimensional film height.

The slip velocity at the porous matrix [29] is given as:

$$
\frac{\partial u}{\partial y}=\frac{\alpha^{\star}}{k^{\frac{1}{2}}} u \text { at } y=0
$$

where $k$ is the porosity of the matrix and $\alpha^{\star}$ is the slip coefficient.

For pressure, the appropriate boundary conditions are

$$
p=0 \text { at } x=0, L \text {; }
$$

where, $L$ is the width of the bearing.

Solving Eq. (8) with the boundary conditions (14) and (15), we obtain the velocity component as

$$
\begin{aligned}
& u=\frac{\frac{\partial}{\partial x}\left(p-\frac{\mu_{0} \bar{\mu}}{2} H^{2}\right)}{\left(\mu-\frac{\rho \alpha^{2} \bar{\mu}}{2} H\right)} \frac{y^{2}}{2} \\
& +\left(U-\frac{\frac{\partial}{\partial x}\left(p-\frac{\mu_{0} \bar{\mu}}{2} H^{2}\right)}{\left(\mu-\frac{\rho \alpha^{2} \bar{\mu}}{2} H\right)} \frac{h^{2}}{2}\right)\left(\frac{1+s y}{1+s h}\right),
\end{aligned}
$$

where $s=-\frac{\alpha^{\star}}{k^{\frac{1}{2}}}$ is the slip parameter.

Using Eqs. (9), (13) and (16), we can get the Reynolds equation in the following form:

$$
\begin{aligned}
& \frac{\partial}{\partial x}\left[\frac { 1 } { 1 2 \mu } \left(12 k l+\frac{h^{3}(4+s h)}{(1+s h)\left(1-\frac{\rho \alpha^{2} \bar{\mu}}{2 \mu} H\right)}\right.\right. \\
& \left.\left.-\frac{3 \rho \alpha^{2} \bar{\mu} k h^{2} s H}{\mu(1+s h)\left(1-\frac{\rho \alpha^{2} \bar{\mu}}{2 \mu} H\right)}\right) \frac{\partial}{\partial x}\left(p-\frac{\mu_{0} \bar{\mu}}{2} H^{2}\right)\right] \\
& =\frac{U}{2} \frac{\partial}{\partial x}\left[\frac{h(2+s h)}{(1+s h)}\right]
\end{aligned}
$$

Integration of equation (18) gives:

$$
\begin{aligned}
& \frac{\partial}{\partial x}\left(p-\frac{\mu_{0} \bar{\mu}}{2} H^{2}\right)= \\
& \frac{\mu^{2}[6 U h(2+s h)+12 A(1+s h)]}{\left[6 k l\left(2 \mu(1+s h)-\rho \alpha^{2} \bar{\mu} H\right)+\mu h^{3}(4+s h)^{2}\right]} \\
& +\frac{\mu H \rho \alpha^{2} \bar{\mu}[6 U h(2+s h)+12 A(1+s h)]}{\left[6 k l\left(2 \mu(1+s h)-\rho \alpha^{2} \bar{\mu} H\right)+\mu h^{3}(4+s h)^{2}\right]^{2}} \times \\
& {\left[3 k h\left(\mu(1+s h)-\frac{\rho \alpha^{2} \bar{\mu}}{2} H\right)-\frac{\mu h^{3}}{2}(4+s h)\right]}
\end{aligned}
$$

\section{Heat Transfer Problem}

Assuming the flow of lubricant thermally active and surfaces thermally inactive [20], the energy equation is simplified to get

$$
\bar{k}\left(\frac{\partial^{2} T}{\partial y^{2}}\right)=-\mu\left(\frac{\partial u}{\partial y}\right)^{2}
$$

Let the viscosity $\mu$ varies exponentially with temperature raised by frictional heat generated by flow i.e.

$$
\mu=\mu_{0} \exp \left[-\beta\left(T_{m}-T_{0}\right)\right]
$$

where

$$
T_{m}=\frac{1}{h} \int_{0}^{h} T d y
$$

In the fluid film region, the boundary conditions for temperature are

$$
T=T_{0} \text { at } y=0 \text { and } y=h
$$




\section{Solution of the Problem}

Introducing the following non-dimensional quantities:

$$
\begin{aligned}
& \bar{x}=\frac{x}{L}, \quad \bar{y}=\frac{y}{h_{0}}, \quad \bar{h}=\frac{h}{h_{0}}, \quad \bar{M}=\frac{\mu}{\mu_{0}}, \quad \bar{A}=\frac{12 A}{U h_{0}}, \\
& u_{0}=\frac{u}{U}, \quad \bar{p}=\frac{p h_{0}^{2}}{\mu U L}, \quad \bar{T}=\frac{T}{T_{0}}, \quad \bar{\alpha}^{2}=\frac{\rho \alpha^{2} \bar{\mu} L}{2 \mu_{0}} \\
& \bar{y}^{2}=\frac{6 k}{h_{0}^{2}}, \quad \bar{\beta}^{3}=\frac{12 k l}{h_{0}^{3}}, \bar{T}_{m}=\frac{T_{m}}{T_{0}}, \quad P_{r}=\frac{\mu_{0} c_{p}}{\bar{k}}, \\
& E=\frac{U^{2}}{c_{p} t_{0}}, \quad B_{0}=\beta t_{0}, \quad \bar{\mu}^{\star}=\frac{\bar{\mu}_{0} \bar{\mu}_{0}^{2} L}{\mu U}, \quad \bar{s}=s h_{0}
\end{aligned}
$$

The dimensionless form of (17), (20), (21) and (22) are:

$$
\begin{gathered}
u_{0}=\frac{\frac{\partial}{\partial \bar{x}}\left(\bar{p}-\frac{\bar{\mu}^{*}}{2} \bar{x}(1-\bar{x})\right)}{\left(1-\bar{\alpha}^{2} \sqrt{\bar{x}(1-\bar{x})}\right)} \frac{\bar{y}^{2}}{2} \\
+\left(1-\frac{\frac{\partial}{\partial \bar{x}}\left(\bar{p}-\frac{\bar{\mu}^{*}}{2} \bar{x}(1-\bar{x})\right)}{\left(1-\bar{\alpha}^{2} \sqrt{\bar{x}(1-\bar{x})}\right)} \frac{\bar{h}^{2}}{2}\right)\left(\frac{1+\bar{s} y}{1+\bar{s} h}\right) \\
\frac{\partial^{2} \bar{T}}{\partial \bar{y}^{2}}=-M P_{r} E\left(\frac{\partial u_{0}}{\partial \bar{y}}\right)^{2} \\
\bar{M}=\exp \left\{-B_{0}\left(\bar{T}_{m}-1\right)\right\} \\
\bar{T}_{m}=\frac{1}{\bar{h}} \int_{0}^{\bar{h}} \bar{T} d \bar{y}
\end{gathered}
$$

Using Eqs. (24), (25) and boundary conditions $\bar{T}=1$ at $\bar{y}=0$ and at $\bar{y}=\bar{h}$, we obtain the non- dimensional temperature field as:

$$
\begin{aligned}
\bar{T}= & 1-M_{0} P_{r} E\left[\bar{y}^{4} \phi^{2}+\frac{\bar{y}^{2} \bar{s}^{2}\left(1-\frac{\bar{h}^{2}}{2} \phi\right)^{2}}{2(1+\bar{s} \bar{h})^{2}}+\frac{\bar{y}^{3} \phi \bar{s}\left(1-\frac{\bar{h}^{2}}{2} \phi\right)}{3(1+\bar{s} \bar{h})}\right] \\
& +\frac{\bar{y} \bar{M} P_{r} E}{\bar{h}}\left[\frac{\bar{h}^{4}}{12} \phi^{2}+\frac{\bar{h}^{2} \bar{s}^{2}\left(1-\frac{h^{2}}{2} \phi\right)^{2}}{2(1+\bar{s} \bar{h})^{2}}+\frac{\bar{h}^{3} \phi \bar{s}\left(1-\frac{h^{2}}{2} \phi\right)}{3(1+\bar{s} \bar{h})}\right],
\end{aligned}
$$

where $\phi=\frac{\frac{\partial}{\partial \bar{x}}\left(\bar{p}-\frac{\tilde{\mu}^{*}}{2} \bar{x}(1-\bar{x})\right)}{\left(1-\bar{\alpha}^{2} \sqrt{\bar{x}(1-\bar{x})}\right)}$.

Putting the value of $\bar{T}$ into equation (27) and on simplifying we obtain the non-dimensional mean temperature as

$$
\begin{aligned}
& \bar{T}_{m}=1+\frac{\bar{M} P_{r} E}{240}\left[6 \bar{h}^{4} \phi^{2}+\frac{5 \bar{h}^{2} \bar{s}^{2}\left(4+\bar{h}^{4} \phi^{2}-4 \bar{h}^{2} \phi\right)}{(1+\bar{s} \bar{h})^{2}}\right. \\
& \left.+\frac{10 \bar{h}^{3} \bar{s} \phi\left(2-\bar{h}^{2} \phi\right)}{(1+\bar{s} \bar{h})}\right]
\end{aligned}
$$

The dimensionless form of equation (19) is

$$
\frac{\partial}{\partial \bar{x}}\left(\bar{p}-\frac{\bar{\mu}^{*}}{2} \bar{x}(1-\bar{x})\right)=\frac{\frac{1}{\bar{s}}(12 \bar{h}+\bar{A})+\bar{h}(6 \bar{h}+\bar{A})}{\frac{1}{\bar{s}}\left(\bar{\beta}^{3}\left(1-\bar{\alpha}^{2} \sqrt{\bar{x}(1-\bar{x})}\right)+4 \bar{h}^{3}\right)+\bar{h}\left(\bar{\beta}^{3}+\bar{h}^{3}\right)}
$$$$
\begin{aligned}
& +\frac{\bar{\alpha}^{2} \bar{h}\left[\frac{1}{\bar{s}}(12 \bar{h}+\bar{A})+\bar{h}(6 \bar{h}+\bar{A})\right]\left[\frac{1}{\bar{s}}\left(\bar{y}^{2}-4 \bar{h}^{2}-\bar{\alpha}^{2} \sqrt{\bar{x}(1-\bar{x})}\right)+\bar{h}\left(\bar{y}^{2}-\bar{h}^{2}\right)\right]}{\left[\frac{1}{\bar{s}}\left(\bar{\beta}^{3}\left(1-\bar{\alpha}^{2} \sqrt{\bar{x}(1-\bar{x})}\right)+4 \bar{h}^{3}\right)+\bar{h}\left(\bar{\beta}^{3}+\bar{h}^{3}\right)\right]^{2}} \times \\
& (\bar{x}(1-\bar{x}))^{\frac{1}{2}}
\end{aligned}
$$

or

$$
\begin{aligned}
& \phi=\frac{\frac{\partial}{\partial \bar{x}}\left(\bar{p}-\frac{\bar{\mu}^{*}}{2} \bar{x}(1-\bar{x})\right)}{\left(1-\bar{\alpha}^{2} \sqrt{\bar{x}(1-\bar{x})}\right)}= \\
& \frac{\frac{1}{\bar{s}}(12 \bar{h}+\bar{A})+\bar{h}(6 \bar{h}+\bar{A})}{\left[\frac{1}{\bar{s}}\left(\bar{\beta}^{3}\left(1-\bar{\alpha}^{2} \sqrt{\bar{x}(1-\bar{x})}\right)+4 \bar{h}^{3}\right)+\bar{h}\left(\bar{\beta}^{3}+\bar{h}^{3}\right)\right]\left(1-\bar{\alpha}^{2} \sqrt{\bar{x}(1-\bar{x})}\right)} \\
& +\frac{\bar{\alpha}^{2} \bar{h}\left[\frac{1}{\bar{s}}(12 \bar{h}+\bar{A})+\bar{h}(6 \bar{h}+\bar{A})\right]\left[\frac{1}{\bar{s}}\left(\bar{y}^{2}-4 \bar{h}^{2}-\bar{\alpha}^{2} \sqrt{\bar{x}(1-\bar{x})}\right)+\bar{h}\left(\bar{y}^{2}-\bar{h}^{2}\right)\right]}{\left[\frac{1}{\bar{s}}\left(\bar{\beta}^{3}\left(1-\bar{\alpha}^{2} \sqrt{\bar{x}(1-\bar{x})}\right)+4 \bar{h}^{3}\right)+\bar{h}\left(\bar{\beta}^{3}+\bar{h}^{3}\right)\right]^{2}\left(1-\bar{\alpha}^{2} \sqrt{\bar{x}(1-\bar{x})}\right)} \times \\
& (\bar{x}(1-\bar{x}))^{\frac{1}{2}}
\end{aligned}
$$

where the film thickness $\bar{h}(\bar{x})$ of the inclined slider bearing is given by $\bar{h}(\bar{x})=a-(a-1) \bar{x}$, and $a=h_{1} / h_{0}, \quad 0 \leq \bar{x} \leq 1$.

In particular, we take the inlet-outlet ratio $a=h_{1} / h_{0}=$ $2 \Rightarrow \bar{h}(\bar{x})=2-\bar{x}=2$.

Now by integrating Eq. (30) and applying the boundary condition $\bar{p}=0$ at $\bar{x}=0$ and $\bar{x}=1$, we obtain the value of as:

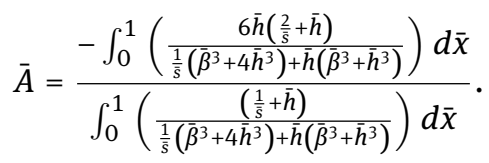

The lifting force or the load carrying capacity is given by:

$$
\bar{W}=\int_{0}^{1} \bar{p} d \bar{x}=-\int_{0}^{1} \bar{x} \frac{d \bar{p}}{d \bar{x}} d \bar{x} .
$$

Using Eqs. (29) and (33), we obtain the nondimensional load capacity of the bearing as:

$$
\begin{aligned}
& \bar{W}=\frac{\bar{\mu}^{\star}}{12}-\int_{0}^{1} \bar{x}\left(\bar{\alpha}^{2} \sqrt{\bar{x}(1-\bar{x})}-1\right) \\
& \sqrt{\frac{1}{6 \bar{h}^{2}+\bar{h}^{4} \bar{s}^{2}+2 \bar{h}^{3} \bar{s}}\left[\frac{240\left(T_{m}-1\right)(1+\bar{s} \bar{h})^{2}}{\bar{h}^{2} \bar{M} P_{r} E}-20 \bar{s}^{2}-20 \phi \bar{h} \bar{s}\right]} d \bar{x}
\end{aligned}
$$

\section{Results and Discussion}

On behalf of the computation and investigations in the present problem, the following results are carried out. 
Fig. 2 shows the variation of mean temperature $\left(\bar{T}_{m}\right)$ w.r.t. the permeability parameter $(\bar{\beta})$. It is noticed that the mean temperature increases exponentially for $\bar{\beta}>0$. So, it is concluded that the porosity of the matrix causes an increase in the mean temperature of the slider bearing. Therefore, for controlling the mean temperature of the slider, the porosity of the matrix should be adjusted suitably.

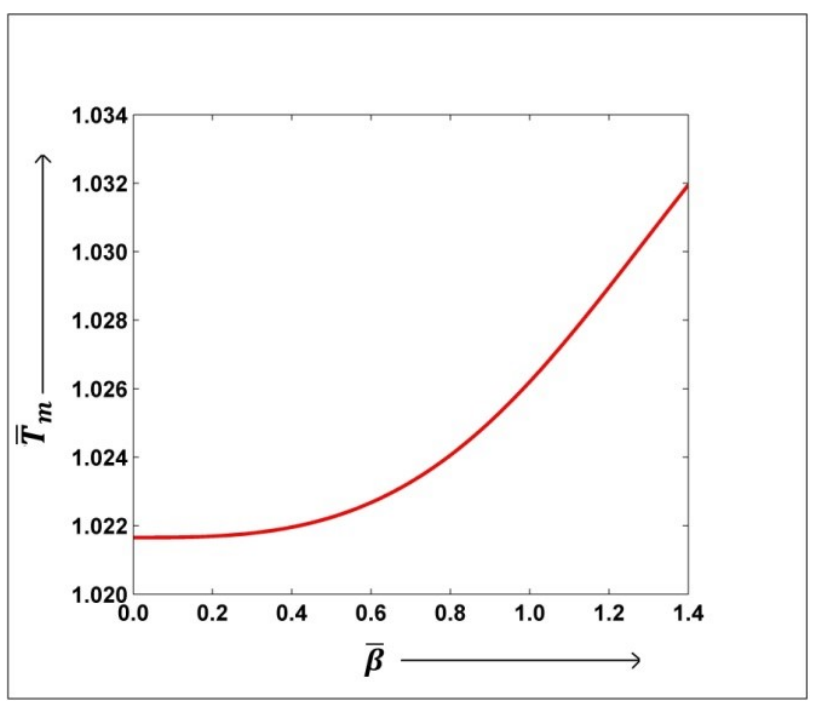

Fig. 2: Mean temperature vs. Permeability parameter at $1 / \bar{s}=$ $1, \bar{\alpha}^{2}=0.8, \bar{y}^{2}=1.2, P_{r} . E=1.2, \bar{x}=0.6$

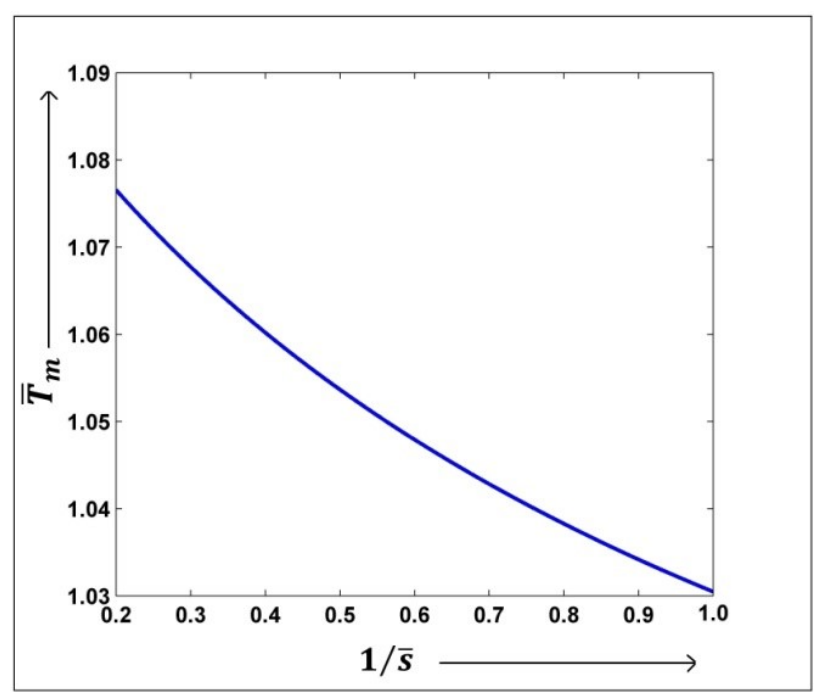

Fig. 3: Mean temperature vs. Slip parameter at $\bar{\beta}=1.3, \bar{\alpha}^{2}=$ $0.8, \bar{y}^{2}=1.2, P_{r} . E=1.2, \bar{x}=0.6$
From Fig. 3 the variation in the mean temperature $\left(\bar{T}_{m}\right)$ w.r.t. the slip parameter $(1 / \bar{s})$, has been observed. The mean temperature decreases with an increase in the slip parameter; this is because the slip velocity reduces the friction between the fluid and the boundary and hence the heat production is reduced which causes a fall in the mean the temperature.

In Fig. 4 and Fig. 5, the behavior of mean temperature $\left(\bar{T}_{m}\right)$ has been noted with thermal $\left(P_{r} . E\right)$ and magnetic parameter $\left(\bar{\mu}^{\star}\right)$ respectively. It is seen that the mean temperature of the bearing increases linearly with thermal as well as magnetic parameter. So, the mean temperature is a linear function of $P_{r} . E$ and $\bar{\mu}^{\star}$.

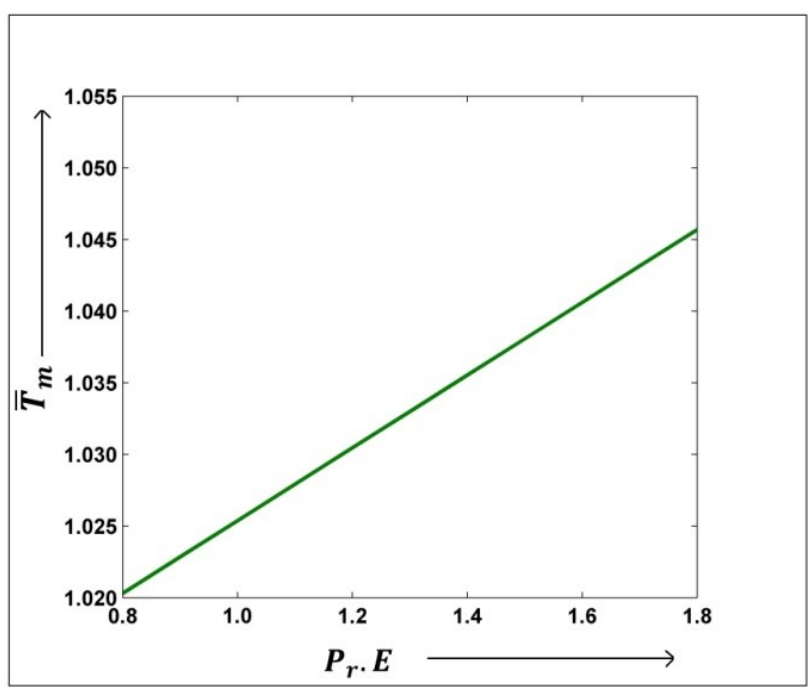

Fig. 4: Mean temperature vs. Thermal parameter at $1 / \bar{s}=1, \bar{\alpha}^{2}=$ $0.8, \bar{\beta}=1.3, \bar{y}^{2}=1.2, \bar{x}=0.6$

The variations of mean temperature $\left(\bar{T}_{m}\right)$ across the fluid film region have been analyzed for different values of the thermal parameter $\left(P_{r} . E\right)$ and the material parameter $\left(\bar{\alpha}^{2}\right)$ in Fig. 6 and Fig. 7 respectively. The area under the mean temperature curve is large for the inner half part $(0 \leq \bar{x} \leq 0.5)$ and negligible for the outer half part $(0.5 \leq \bar{x} \leq 1)$ of the slider. Therefore, the width of the thermal boundary layer is quite large or the heat dissipation is very slow in the inner half part as compared to the outer half part of the bearing. From Fig. 7, it is observed that the width of the thermal boundary layer is much affected by the material parameter $\left(\bar{\alpha}^{2}\right)$ in the inner half part of the slider. So, for a desirable rate of heat transfer, the value of the material parameter $\left(\bar{\alpha}^{2}\right)$ should be adjusted accordingly.

Fig. 8 reveals the variation of mean temperature $\left(\bar{T}_{m}\right)$ with slip parameter $(1 / \bar{s})$ for different values of the ma- 


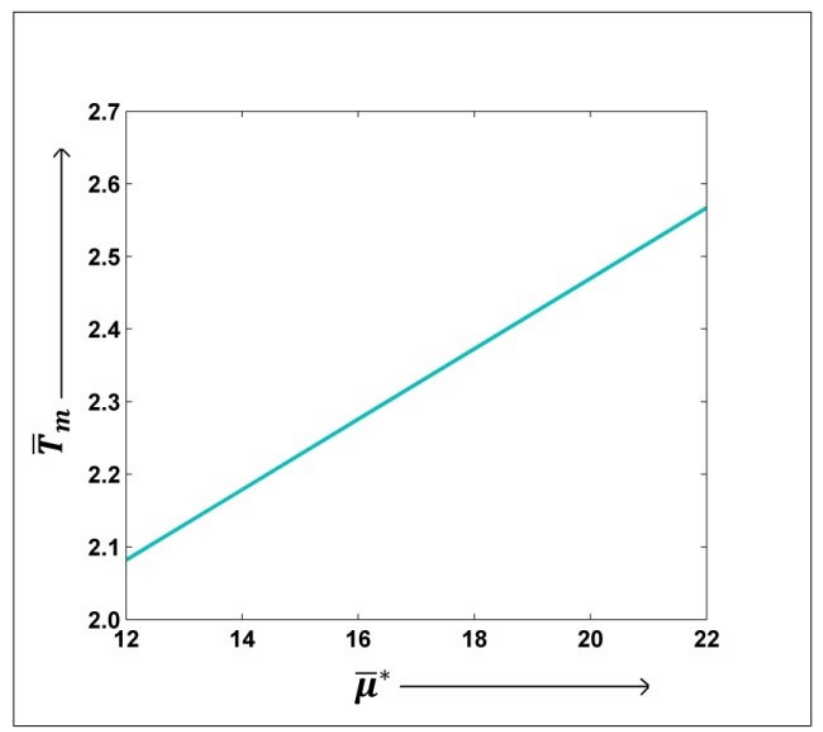

Fig. 5: Mean temperature vs. Magnetic parameter at $1 / \bar{s}=1, \bar{\alpha}^{2}=$ $0.8, \bar{\beta}=1.3, \bar{y}^{2}=1.2 P_{r} . x=1.2, \bar{x}=0.6$

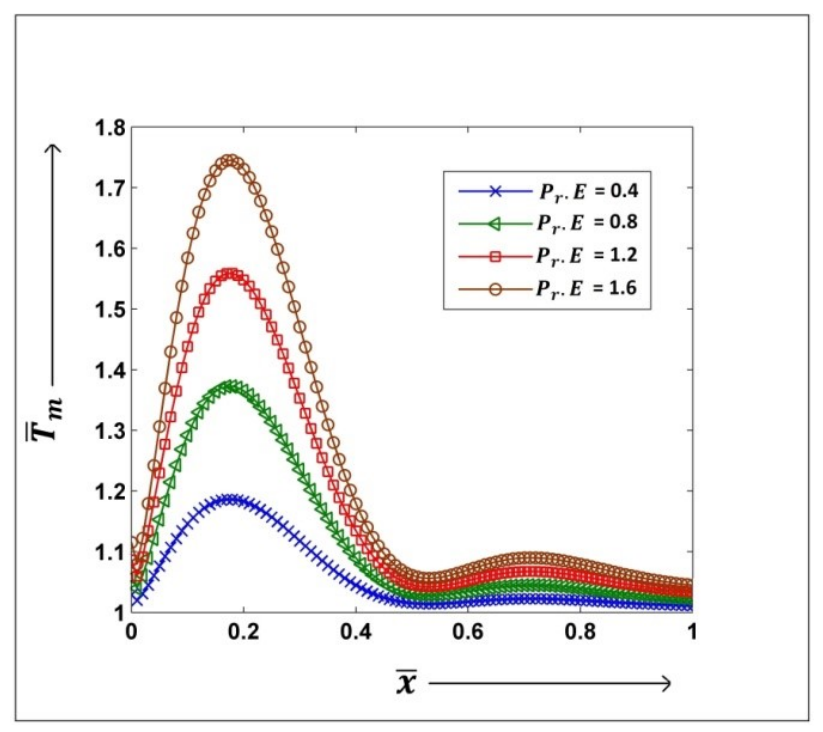

Fig. 6: Mean temperature vs. bearing length for various values of the thermal parameter at $1 / \bar{s}=0.5, \bar{\alpha}^{2}=1.2, \bar{\beta}=1.3, \bar{y}^{2}=1.2$

terial parameter $\left(\bar{\alpha}^{2}\right)$. It is seen that for a small value of slip, i.e. $1 / \bar{s} \leq 0.3$, the material parameter doesn't affect the mean temperature but for a large value of slip, i.e. $1 / \bar{s}>0.3$, it has a notable effect on mean temperature. Therefore, for $1 / \bar{s}>0.3$, the material parameter should be adjusted according to the boundary slip.

The variations of the lifting force $(\bar{W})$ versus permeability parameter $(\bar{\beta})$ under the influence of material parameter $\left(\bar{\alpha}^{2}\right)$ have been observed in Fig. 9. It is noted that the influence of the permeability parameter is not remarkable for a smaller value of the material parameter but for

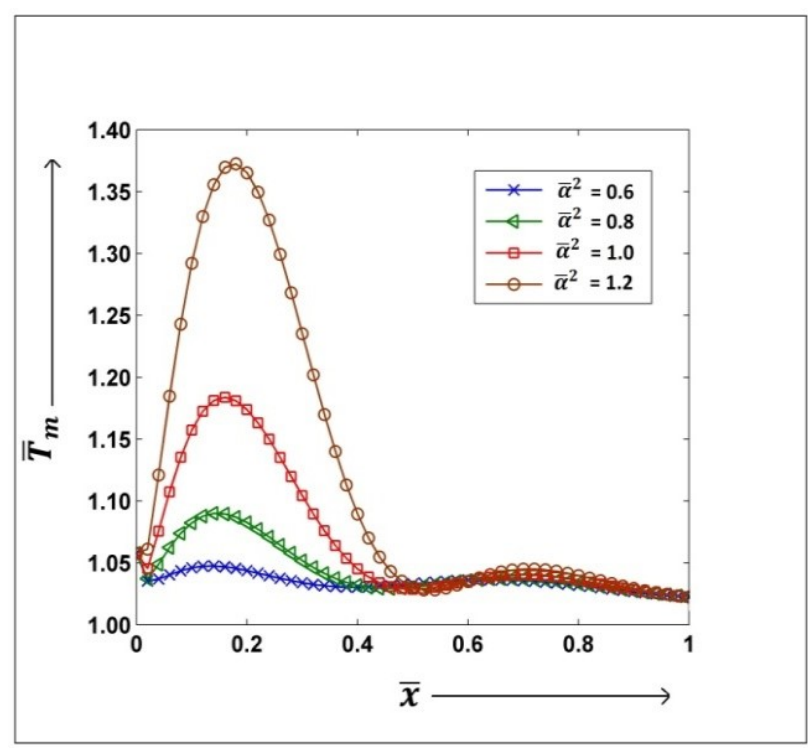

Fig. 7: Mean temperature vs. bearing length for various values of the material parameter at $1 / \bar{s}=0.5, \bar{\beta}=1.3, \bar{y}^{2}=1.2, P_{r} . E=$ 0.8

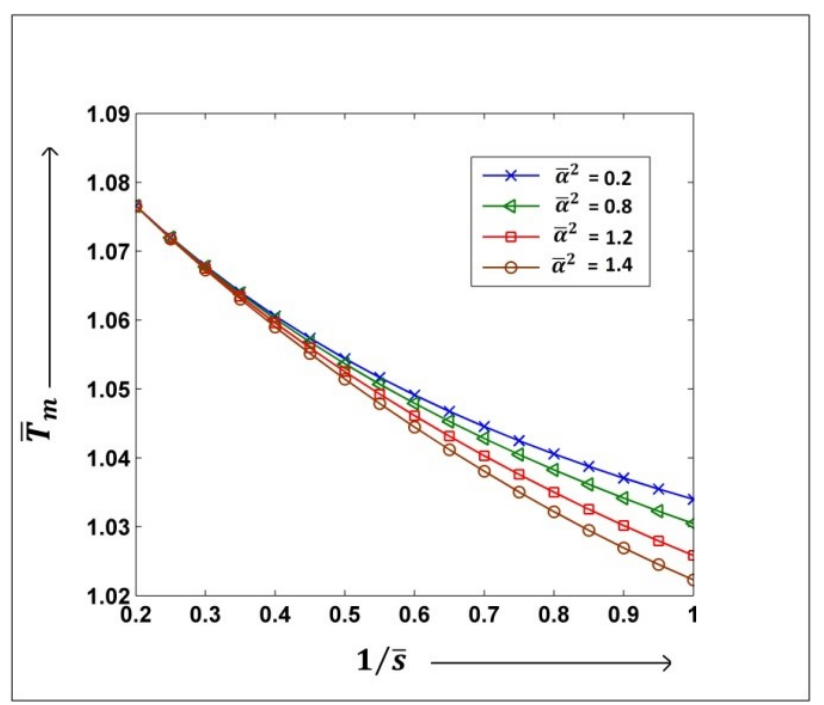

Fig. 8: Mean temperature vs. Slip parameter for different values of the material parameter at $\bar{\beta}=1.3, \bar{y}^{2}=1.2, P_{r} . E=1.2, \bar{x}=0.6$

larger values of the material parameter, the load capacity decreases with an increase in the permeability parameter.

The behavior of the lifting force w.r.t. the thermal parameter $\left(P_{r} . E\right)$ for different values of the magnetic parameter $\left(\bar{\mu}^{\star}\right)$ has been plotted in Fig. 10. It is observed that the lifting force decreases with an increase in the thermal parameter. It is because the enhancement in the thermal parameter causes an increase in the mean temperature. Due to this the heat dissipation becomes slow which results in a fall in the lifting force or the load carrying capacity. Some- 


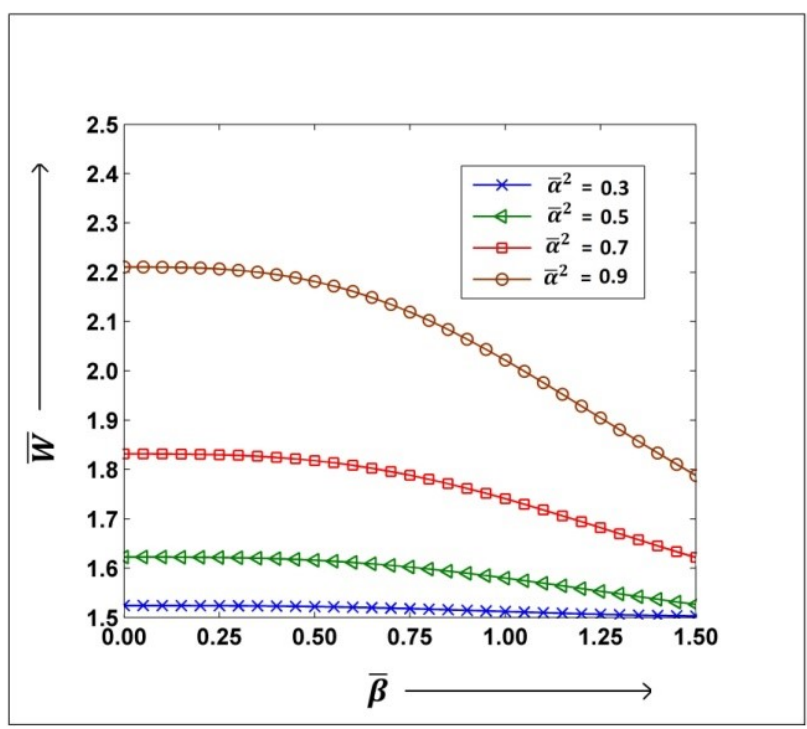

Fig. 9: Lifting force vs. Permeability parameter for different values of the magnetic parameter at $1 / \bar{s}=1.2, \bar{y}^{2}=1.2, P_{r} \cdot E=1.2, \bar{\mu}^{\star}=$ 12

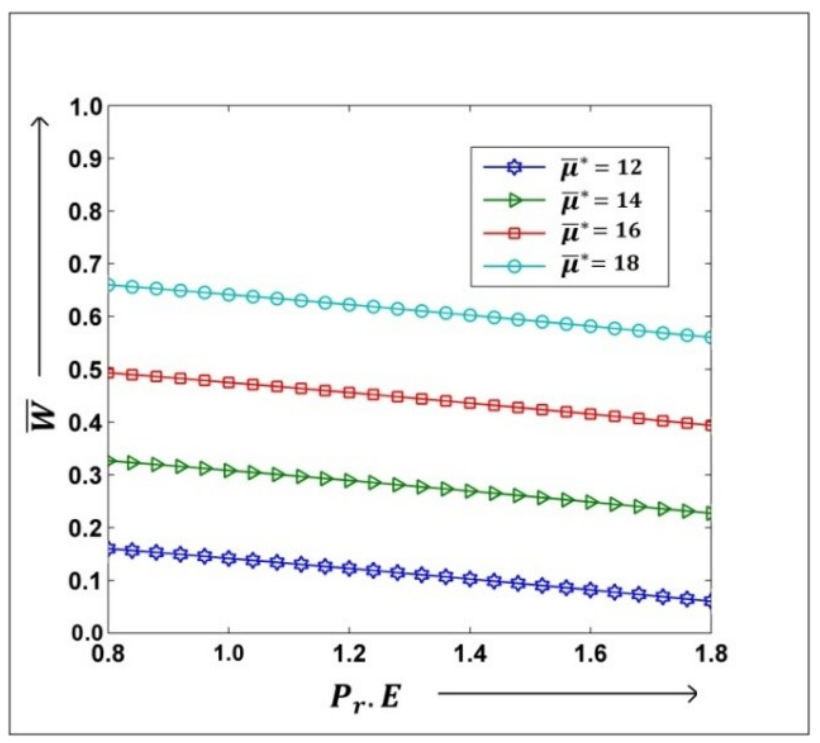

Fig. 10: Lifting force vs. Thermal parameter for different values of the magnetic parameter at $1 / \bar{s}=1, \bar{\alpha}^{2}=1, \bar{\beta}=1.3, \bar{y}^{2}=1.2$

times the bearing may break due to overheating between the pads.

Fig. 11 reveals the variations in the lifting force $(\bar{W})$ w.r.t. the inlet-outlet ratio $(a)$ for different values of the magnetic parameter $\left(\bar{\mu}^{*}\right)$. It is noted that the lifting force enhances with an increase in the inlet-outlet ratio. Therefore, the angle of inclination of the inclined pad has also an impact on the performance of the slider bearing. In Fig. 10 and 11 it is also noted that the magnetic parameter boosts the lifting force of the slider. It is because the mag-

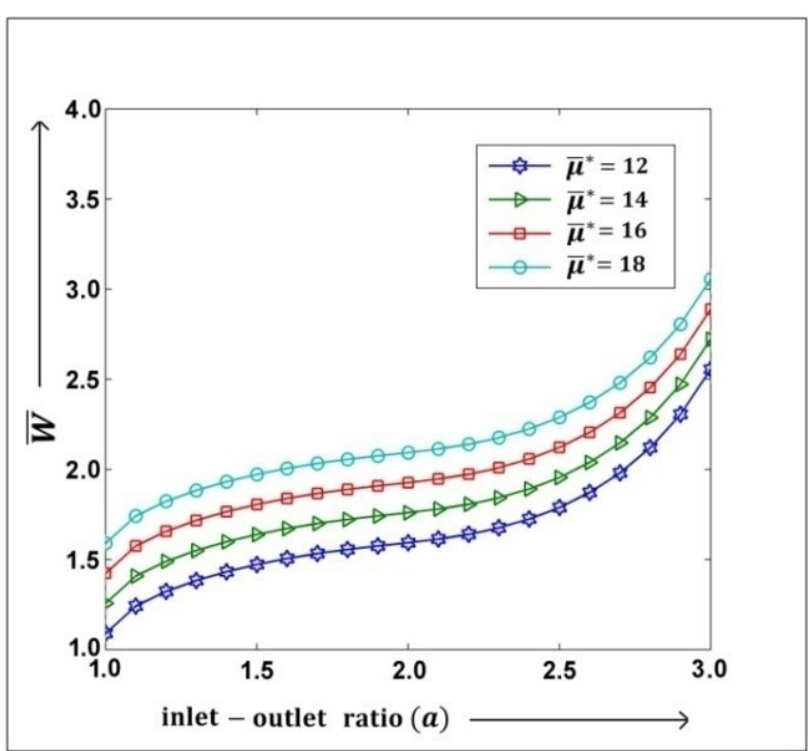

Fig. 11: Lifting force vs. Inlet-outlet ratio for different values of the magnetic parameter at $1 / \bar{s}=1, \bar{\alpha}^{2}=0.25, \bar{\beta}=1.3, \bar{y}^{2}=$ 1.2, $P_{r} . E=1.2$

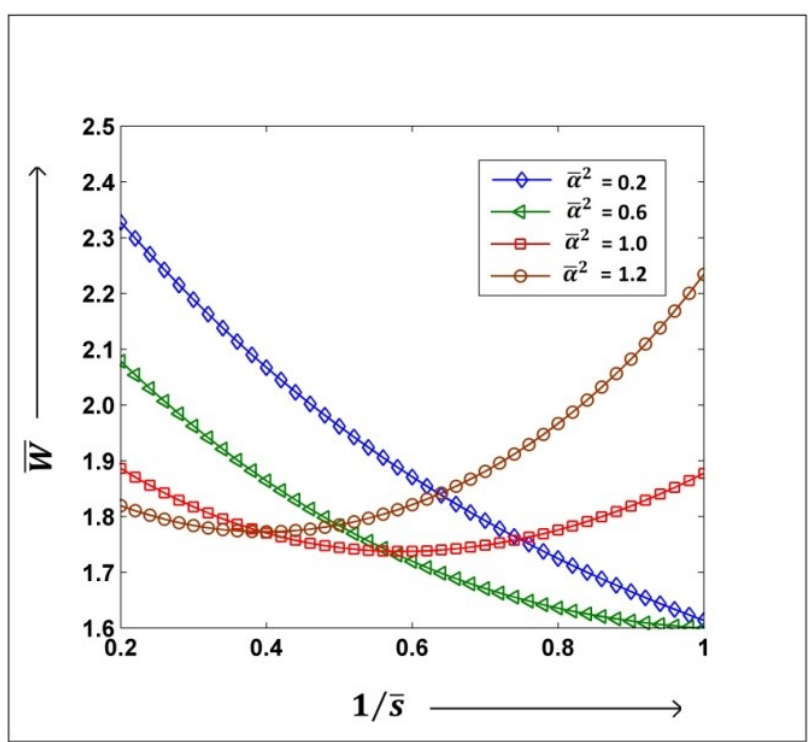

Fig. 12: Lifting force vs. slip parameter for different values of the magnetic parameter at $\bar{\beta}=1.3, \bar{y}^{2}=1.2, P_{r} . E=1.2, \bar{\mu}^{\star}=12$

netization increases the viscosity of the lubricant, which results in an increase in the pressure and consequently, the lifting force.

In Fig. 12, the variations in the lifting force with the slip parameter have been noted. It is observed that for smaller values of the material parameter, i.e. $\bar{\alpha}^{2} \leq 0.6$, the lifting force decreases with an increase in the slip parameter but the trend is reversed for larger values of the material parameter, i.e. $\bar{\alpha}^{2}>0.6$. Therefore, the performance of the bearing in terms of the lifting force w.r.t. the slip parameter 
depends on the material properties like density, magnetic susceptibility, the coefficient of viscosity, bearing width, slip region, surface texture, etc.

Validation of the Results: In Figures 2-5, the trends of the graphs are similar to Singh and Ahmad [20] and the deviations in the values of mean temperature and the lifting force is due to the additional term of the Co rotational derivative of magnetization. If we remove the effect of this term, the results obtained are similar to Singh and Ahmad [20]. In Figure 9 the trend of the graph is also similar to Singh and Ahmad [20] for small values of the material parameter.

For larger values of the material parameter in Fig. 12, the trend of the graph shows that the effect of boundary slip is same as obtained by Q. Lin [28].

Further, in Figure 11, it is noted that the load capacity or lifting force enhances with an increase in the inletoutlet ratio. The same result is also obtained by Ram et al. [21], and hence the results in the present paper validate the existing results.

\section{Conclusions}

- Jenkins Model for lubrication has been analyzed with slip and thermal effects using magnetic nanofluid as the lubricant. The present work recommends that the mean temperature has been accelerated by the permeability parameter and decelerated by slip velocity. Also, the mean temperature increases linearly with the thermal and the magnetic parameter.

- The heat dissipation or the cooling is quite slow in the inner part of the slider as compared to its outer part. The material parameter also has a notable effect on the thermal boundary layer so for desirable heat transfer; its value should be adjusted accordingly.

- $\quad$ For small values of the material parameter, the permeability parameter does not have much effect on the lifting force, but for a large value of the material parameter, the lifting force decreases with an increase in the permeability parameter. Therefore for large values of the material parameter, the permeability i.e., the porosity of the matrix should be adjusted suitably to get the maximum load capacity.
- With an increase in the thermal parameter, the lifting force decreases due to overheating. Therefore, the value of the thermal parameter, i.e., Prandtl number and Eckert number should be adjusted in such a way that the loss due to heat can be minimized and tribological performance of the bearing can be improved.

- $\quad$ For a large value of the material parameter, i.e. $\bar{\alpha}^{2}>$ 0.6 , the lifting forcehas a dual nature with the slip parameter Firstly, the load decreases with increase in the slip parameter, and the trend is reversed after an optimum value of the slip parameter. Therefore, proper slip conditions which depend on the material properties of the bearing are required to get the better tribological performance of the bearing in terms of the lifting force.

Finally, it is suggested that while designing a slider bearing, special care has to be taken about the porosity of the matrix, material properties, slip boundary conditions, inlet-out ratio depending on the pad length, heat dissipation rate, etc. for its better tribological performance in terms of the lifting force.

\section{Nomenclature}

a Inlet-outlet ratio

$B_{0}$ The non-dimensional coefficient of temperature

$H$ The strength of the external magnetic field

$h$ Dimensional film height

$h_{0}$ Minimum film thickness

$h_{1}$ Maximum film thickness

$\bar{h}$ Non-dimensional film height

$k$ The porosity of the porous matrix

$\bar{k}$ Thermal Conductivity

$l$ Bearing wall thickness

$L$ Bearing width

$\tilde{M}$ Magnetization vector

$M^{\star}$ Co-rotational derivative of $\tilde{M}$

$M$ The magnitude of the magnetization vector

$\bar{M}$ The non-dimensional coefficient of viscosity

$p$ Fluid pressure

$\bar{p}$ Non-dimensional fluid pressure

$P_{r}$ Prandtl number

$1 / \bar{s}$ Non-dimensional slip parameter

$s$ Slip parameter

$t$ The temperature of the fluid

$t_{0}$ Ambient temperature 
$t_{m}$ Mean temperature across the film thickness

$T_{m}$ Non-dimensional mean temperature

$T$ Non-dimensional temperature field

$(x, y)$ Cartesian coordinates

$\bar{x}$ Non-dimensional $\mathrm{x}$-coordinate

$u$ Velocity component along the $\mathrm{x}$-axis

$u_{0}$ Non-dimensional velocity component along the $x$-axis

$U$ Uniform sliding velocity component along the $\mathrm{x}$-axis

$\vec{V}$ Fluid velocity

$\bar{W}$ Non-dimensional load capacity or lifting force

$\alpha^{\star}$ Slip constant

$\alpha^{2}$ Material parameter

$\bar{\alpha}^{2}$ Non-dimensional material parameter

$\beta$ Coefficient of temperature

$\bar{\beta}$ Non-dimensional permeability parameter

$\mu$ Coefficient of viscosity

$\mu_{0}$ Free space permeability

$\bar{\mu}^{\star}$ Non-dimensional magnetic parameter

$\bar{\mu}$ Magnetic susceptibility

\section{References}

[1] Esfandiary M, Mehmandoust B, Karimipour A, Pakravan HA. Natural convection of Al203-water nanofluid in an inclined enclosure with the effects of slip velocity mechanisms: Brownian motion and thermophoresis phenomenon. International Journal of Thermal Sciences 2016, 105, 137-58.

[2] Karimipour A, Nezhad AH, D’Orazio A, Esfe MH, Safaei MR, Shirani E. Simulation of copper-water nanofluid in a microchannel in slip flow regime using the lattice Boltzmann method. European Journal of Mechanics-B/Fluids 2015, 49, 89-99.

[3] Karimipour A, Esfe MH, Safaei MR, Semiromi DT, Kazi SN. Mixed convection of copper-water nanofluid in a shallow inclined lid-driven cavity using the lattice Boltzmann method. Physica A: Statistical Mechanics and its Applications 2014, 402, 150-68.

[4] Esfe MH, Esforjani SSM, Akbari M, Karimipour A. Mixedconvection flow in a lid-driven square cavity filled with a nanofluid with variable properties: effect of the nanoparticle diameter and of the position of a hot obstacle. Heat Transfer Research 2014, 45(6).

[5] Karimipour A, Nezhad AH, Behzadmehr A, Alikhani S, Abedini E. Periodic mixed convection of a nanofluid in a cavity with top lid sinusoidal motion. Proceedings of the Institution of Mechanical Engineers, Part C: Journal of Mechanical Engineering Science 2011, 225(9), 2149-60.

[6] Karimipour A. New correlation for Nusselt number of nanofluid with Ag/Al203/Cu nanoparticles in a microchannel considering slip velocity and temperature jump by using lattice Boltzmann method. International Journal of Thermal Sciences 2015, 91, 146-56.

[7] Pal AK, Das NC, Choudhury P. A study of load caacity of finite slider bearings lubricated with couple stress fluids considering thermal effects. Indian Journal of Pure \& Applied Mathematics
2002, 33(10), 1529-40.

[8] Sinha P, Chandra P, Bhartiya S. Analysis of a non-constant gap externally pressurized conical bearing with temperature and pressure dependent viscosity. Proceedings of the Institution of Mechanical Engineers, Part C: Journal of Mechanical Engineering Science 2000, 214(5), 699-710.

[9] Ram P, Joshi VK, Sharma K, Walia M, Yadav N. Variable viscosity effects on time-dependent magnetic nanofluid flow past a stretchable rotating plate. Open Physics 2016, 14(1), 651-58.

[10] Joshi VK, Ram P, Sharma RK, Tripathi D. Porosity effect on the boundary layer Bodewadt flow of a magnetic nanofluid in the presence of geothermal viscosity. The European Physical Journal Plus 2017, 132(6), 254.

[11] Ram P, Singh H, Kumar R, Kumar V, Joshi VK. Free convective boundary layer flow of radiating and reacting MHD fluid past a cosinusoidally fluctuating heated plate. International Journal of Applied and Computational Mathematics 2017, 3(1), 1-34.

[12] Ram P, Joshi VK, Makinde OD. Unsteady convective flow of hydrocarbon magnetite nano-suspension in the presence of stretching effects. Defect and Diffusion Forum 2017, 377, 155 65.

[13] Verma PDS, Ram P. On the low-Reynolds number magnetic fluid flow in a helical pipe. International Journal of Engineering Science 1993, 31(2), 229-39.

[14] Ram P, Bhandari A. Flow characteristics of revolving ferrofluid with variable viscosity in aporous medium in the presence of stationary disk. Fluid Dyn. Mater. Process 2012, 8(4), 437-52.

[15] Ram P, Kumar V. Ferrofluid flow with magnetic field-dependent viscosity due to rotating disk in porous medium. International Journal of Applied Mechanics 2012, 4(4), 1250041.

[16] Ellahi R, Tariq MH, Hassan M, Vafai K. On boundary layer nanoferro liquid flow under the influence of low oscillating stretchable rotating disk. Journal of Molecular Liquids 2017, 229, 339 . 45.

[17] Li X, Shinshi T, Hijikata W, Morimoto Y. Development of asuspension type sliding planar motion table using magnetic fluid lubrication. Review of Scientific Instruments 2016, 87(6), 065003.

[18] Huang W, Wang X. Ferrofluids lubrication: a status report. Lubrication Science 2016, 28(1), 3-26.

[19] Ram P, Verma PDS. Ferrofluid lubrication in porous inclined slider bearing. Indian Journal of Pure and Applied Mathematics 1999, 30(12), 1273-82.

[20] Singh JP, Ahmad N. Analysis of a porous-inclined slider bearing lubricated with magnetic fluid considering thermal effects with slip velocity. Journal of the Brazilian Society of Mechanical Sciences and Engineering 2011, 33(3), 351-56.

[21] Ram P, Kumar A, Makinde OD, Kumar P, Joshi VK. Performance analysis of magnetite nano-suspension based porous slider bearing with varying inclination and slip parameter. Diffusion Foundations 2017, 11, 11-21.

[22] Patel JR. Numerical modeling of Jenkins model based ferrofluid lubrication squeeze film performance in rough curved annular plates under the presence of slip velocity. Facta Universitatis, Series: Mathematics and Informatics 2016, 31(1), 11-31.

[23] Laghrabli S, El Khlifi M, Nabhani M, Bou-Saïd B. Ferrofluid lubrication of finite journal bearings using Jenkins model. Lubrication Science 2017, 29(7), 441-54.

[24] Patel JR, Deheri G. Shliomis model-based magnetic squeeze film in rotating rough curved circular plates: a comparison of 
two different porous structures. International Journal of Computational Materials Science and Surface Engineering 2014, 6(1), 29-49.

[25] Laghrabli S, El Khlifi M, Nabhani M, Bou-Saïd B. Static characteristics of ferrofluid finite journal bearing considering rotational viscosity effect, Lubrication Science 2017, 29(4), 203-26.

[26] Ram P, Sharma K, Bhandari A. Effect of porosity on ferrofluid flow with rotating disk. International Journal of Applied Mathematics and Mechanics 2010, 6(16), 67-76.

[27] Shah RC, Tripathi SR, Bhat MV. Magnetic fluid based squeeze film between porous annular curved plates with the effect of rotational inertia. Pramana 2002, 58, 545-50.
[28] Lin Q, Li B. Comparison of the influences of surface texture and boundary slip on tribological performances. Mathematical Problems in Engineering 2015, 2015.

[29] Patel KC, Gupta JL. Hydrodynamic lubrication of a porous slider bearing with slip velocity. Wear 1983, 85(3), 309-17.

[30] Lin Q, Bao Q, Li K, Khonsari MM, Zhao H. An investigation into the transient behavior of journal bearing with surface texture based on fluid-structure interaction approach. Tribology International 2018, 118, 246-55.

[31] Lin Q, Wei Z, Zhang Y, Wang N. Effects of the slip surface on the tribological performances of high-speed hybrid journal bearings. Proceedings of the Institution of Mechanical Engineers, Part J: Journal of Engineering Tribology 2016, 230(9), 1149-56. 